\begin{tabular}{|l|l|}
\hline $\begin{array}{l}\text { Postprint } \\
\text { Version }\end{array}$ & 1.0 \\
\hline Journal website & http://ps.psychiatryonline.org/article.aspx?articleid=1487316 \\
\hline Pubmed link & $\underline{\text { http://www.ncbi.nlm.nih.gov/pubmed/23280464 }}$ \\
\hline DOI & $10.1176 /$ appi.ps.201100541 \\
\hline
\end{tabular}

This is a NIVEL certified Post Print, more info at http://www.nivel.eu

\title{
Mental Health Treatment Provided by Primary Care Psychologists in the Netherlands
}

Peter F. M. VerhaAK, Ph.D. Hans Kamsma, M.Sc. AnneKe Van Der Niet, M.Sc.

\begin{abstract}
Objective: The study examined outcomes of clients treated by primary care psychologists in 2010 in the Netherlands. Methods: Data for 55,067 clients treated by 613 primary care psychologists were analyzed for three outcomes: regular conclusion of treatment, improvement of $>10$ points on the Global Assessment of Functioning (GAF), and treatment conclusion in eight or fewer sessions. Logistic regression analyses examined relationships between client characteristics and outcome. Results: For $73 \%$ of clients, treatment reached a regular conclusion, and for $71 \%$ of these clients treatment was concluded in eight or fewer sessions. An improvement of $>10$ GAF points was achieved by $47 \%$. Women, clients with more years of education, those born in the Netherlands, and those with adjustment problems or child problems were more likely to have a regular treatment conclusion and an improvement of $>10$ GAF points.

Conclusions: Clients with psychopathology of a low or moderate severity were treated successfully by primary care psychologists.
\end{abstract}

Most mental health problems are treated by primary care providers, mainly family physicians $(1,2)$, and mainly with medication (3). Many persons with mental health problems, however, have concerns about drug treatment and prefer counseling (4). Research has shown that women, patients with previous counseling experience, and patients with concerns about medication prefer counseling to medication (5).

Provision of short-term psychological interventions might be a solution in these cases. Such interventions are effective in primary care (6). Counseling in primary care has been shown to be more effective than care as usual and was viewed as a useful addition to care provided by the family physician (7). Counseling has been shown to reduce the number of contacts with the family physician, the number of prescriptions, and the number of mental health referrals (8).

Since 2008 the Dutch health insurance system has reimbursed providers for shortterm psychological interventions and counseling (up to eight sessions). Such "primary care psychological treatment" can be provided by psychologists with a postgraduate qualification as a "health care psychologist.” Primary care psychologists are members of the National Association of Primary Care 
Psychologists (LVE in Dutch). They are independent professionals based in the community, and they work in close collaboration with general practitioners.

They provide short-term treatments with a generalist approach, mostly based on cognitive-behavioral therapy or client-centered therapy.

Approximately 1,000 LVE members offer their services (or about one per 16,000 population). In addition, it is estimated that another 500 psychologists with comparable qualifications who are not LVE members offer the same services. Traditionally, patients in the Netherlands with mental health problems who are not treated by a general practitioner are referred to specialized mental health care. From 2000 to 2009, a substantial increase (170\%) was noted in use of specialized mental health care in the Netherlands (9). A considerable proportion of patients referred to such care turned out to have adjustment disorders or not to have a DSM-IV axis I diagnosis (most had psychosocial problems, with an axis IV diagnosis). Since 2012, under new insurance measures, no reimbursement is provided for specialized mental health care for adjustment disorders or psychosocial problems.

Is the deployment of primary care psychologists a feasible way to meet the needs of these individuals? This question goes beyond the Dutch situation, because the need for accessible treatment, preferably in primary care, is experienced worldwide (10). To answer this question, a better understanding is needed of the clients seen by primary care psychologists and the types of clients for whom their interventions are most successful. The purpose of this study was to evaluate whether treatment by primary care psychologists was an effective substitute for specialized mental health care - that is, whether the treatment of patients who would formerly have been seen in specialized settings was successful and whether effective treatment could be provided in a limited number of sessions. We examined client characteristics or requests for help that were associated with regular conclusion of treatment, a substantial improvement in functioning, and regular treatment conclusion in eight or fewer sessions.

\section{Methods}

Primary care psychologists in the Netherlands are obliged to provide anonymous data on all cases closed during the past year. In accordance with Dutch privacy law, patients are informed about this requirement by their therapist and may opt out, in which case their data are not included in the anonymous upload. Data are uploaded in a fixed format that is identical for all primary care psychologists. From January to March 2011, they completed their upload of all cases closed in 2010. The following outcome data are available: conclusion of treatment (premature conclusion, referral to another mental health care provider, or regular conclusion), Global Assessment of Functioning (GAF) score at the first and the final contacts, and the number of sessions. Available data on client characteristics include sex, age, marital status, education, ethnicity, and employment status. Primary care psychologists use a list of several categories in which to code requests for help. They are also required to categorize diagnoses according to the main categories on the five axes of DSM-IV. Data were analyzed by using Stata, version 11.0. Outcome measures were regular conclusion of treatment (versus interrupted treatment or referral), improvement of .10 GAF points (versus less improvement, no improvement, or a decrease in points), and treatment conclusion in eight sessions or fewer (versus treatment lasting more 
than eight sessions). In logistic regression analysis of improvement in GAF scores, the initial GAF score was included as a correction factor.

Multivariate logistic regression analysis was conducted with all patient characteristics as determinants. A request for help in a single area was a categorical determinant, with all other requests for help as the reference.

If a relationship is reported in the text as significant, the analysis controlled for other possible determinants and for the initial GAF score when GAF was the outcome measure. Because of the large number of cases, the level of significance was set at .001 .

\section{RESULTS}

We analyzed data for 55,067 clients, who were treated by 613 primary care psychologists. [Three tables presenting descriptive statistics for client characteristics are available in an online supplement to this report.] Most clients were female (65\%), 18 to 50 years old (70\%), married or cohabitating or divorced (65\%), and born in the Netherlands (92\%). The most common requests for help were in the areas of interpersonal problems (23\%) and adjustment problems (21\%), followed by depressive feelings (13\%), anxious or nervous feelings (12\%), problems at work or school (9\%), and problems of children under age 18 (8\%).

The primary care psychologists provided DSM-IV diagnoses as follows: axis I, 68\%; axis II personality disorder, 5\%; and axis IV psychosocial problem, 78\%. For 64\% of clients, axis I or II diagnoses co-occurred with an axis IV psychosocial problem. Adjustment disorder (23\%) was the most frequent axis I diagnosis, followed by mood disorder (20\%), and anxiety disorder (15\%). Most psychosocial problems could be categorized as problems in the primary support groups (42\%), problems with work (17\%), and problems within the social context (9\%), reflecting the large proportion of requests for help for interpersonal problems and problems at work. Seventy-three percent of the cases ended in a regularmanner (the clients attended all scheduled appointments). In 13\% of cases, clients stopped attending appointments or terminated therapy. In 14\% of cases, clients were referred to another professional, in most cases to specialized mental health care. Forty-seven percent of the clients showed an improvement of .10 GAF points, and 73\% were treated in eight or fewer sessions. When we limited the analysis to those with a regular treatment conclusion, $56 \%$ improved .10 GAF points and 71\% were treated in eight or fewer sessions. Table 1 presents results of the multivariate logistic regression analysis of three outcomes - regular conclusion of treatment, regular conclusion with major improvement, and regular conclusion in eight sessions or fewer. Several groups were more likely to have had a regular conclusion of treatment and an improvement of .10 GAF points: women, married or cohabiting persons, those with more years of education, and those born in the Netherlands. Compared with clients age 61 and older, children were less likely to have had a regular treatment conclusion. However, children were more likely than all other age groups to have shown an improvement of .10 GAF points. The likelihood of a regular conclusion in eight or fewer sessions was higher among men than among women and among persons with fewer years of education. Premature termination of treatment was more likely among clients age 18-50, persons with fewer years of education, those not born in the Netherlands, and the unemployed (data not shown). 
The analysis compared clients with a particular help request and clients with all other requests (reference category). Clients with adjustment problems had the highest likelihood of a regular treatment conclusion, an improvement of .10 GAF points, and conclusion in eight or fewer sessions. Clients with requests for and conclusion in eight or fewer sessions. Clients with requests for help with child problems were more likely than clients with other problems to have a regular treatment conclusion and to experience improvement of .10 GAF points; however, their treatment was less likely to end in eight or fewer sessions. Clients with feelings of anxiety and with identity problems were more likely than clients with other problems to experience improvement of .10 GAF points; however, clients with feelings of anxiety were less likely to have a regular treatment conclusion.

\section{[TABLE 1]}

Clients with interpersonal problems were less likely than those with other problems to experience an improvement of .10 GAF points, but they were more likely to conclude treatment in eight or fewer sessions.

Clients in the category of problems with sleeping, eating, sexual, or impulse problems were less likely to have a regular treatment conclusion.

For this group, treatments ended prematurely because clients did not attend or decided not to continue with treatment. Clients in this group and clients with depressive feelings were referred relatively often to specialty care (data not shown).

\section{Discussion}

This study found that two-thirds of clients treated by primary care psychologists in the Netherlands had psychopathology — that is, axis I DSM-IV diagnoses. Although this was not an effectiveness study, we conclude from our naturalistic data set that most clients improved in functioning in eight or fewer sessions and experienced sufficient improvement to reach an agreement with their provider to conclude treatment.

Compared with clients who had other types of problems, those with adjustment problems were the most likely to have positive outcomes in all three areas: a regular treatment conclusion, an improvement of .10 GAF points, and treatment conclusion in eight or fewer sessions. Notably, the category of adjustment problems is one for which specialized care is no longer reimbursed by the Dutch insurance system. Clients who requested help for feelings of anxiety, identity problems, and child problems were also more likely than those with other problems to experience an improvement of .10 GAF points.

Women were more likely than men to experience an improvement of.10 GAF points, and clients with more years of education were more likely than those with less education to experience this level of improvement.

Previous studies of counseling and other kinds of psychological treatment have reported similar findings $(5,7)$.

Treatment led to substantial improvement for clients with a wide range of problems: adjustment disorder, anxiety, identity problems, and child problems. In addition, clients with a range of problems were able to conclude treatment in eight or fewer sessions. In specialized mental health care, an average treatment duration of 19 sessions has been reported after referral by a general practitioner; therefore, 
treatment by a primary care psychologist can be considered a shortterm intervention because it does not normally exceed 13 sessions (11).

A major strength of our analysis is the use of a large and representative data set representing the caseloads of most professionals accredited by the National Association of Primary Care Psychologists. Psychologists who are not members of the association work under the same insurance rules and restrictions, and we do not believe that their treatment and clients differ from those in our study. Therefore, we believe our results are a fair representation of the approximately 200,000

psychological treatments provided annually in the Netherlands.

One limitation was our inability to check the reliability or validity of client data provided by the psychologists.

During upload the data were checked only for "logical" errors and suspicious outcomes; psychologists uploading data were given feedback and an opportunity for correction.

Another limitation is that the clinician rated GAF score was the only available outcome indicator. Although GAF scores have been reported to be valid and reliable for research purposes (12), some clinicians have raised doubts about the scores' usefulness in clinical practice. We used GAF scores mainly to compare various groups and assumed that the weakness of the instrument would have been equally distributed over the groups.

\section{CONCLUSIONS}

It is critical that clients for whom specialized mental health care is not indicated obtain treatment in primary care. The findings of this study indicate that primary care psychologists can contribute to a successful shift of patients with adjustment disorders and psychosocial problems from specialized care to primary care. Nevertheless, in June 2012, further changes in mental health care policies in the Netherlands have limited care provided by primary care psychologists to individuals with a DSM-IV diagnosis. Patients with adjustment disorders and psychosocial problems are now dependent on the help of primary care physicians, supported by mental health nurse-assistants.

\section{ACKNOWLEDGMENTS AND DISCLOSURES}

The authors report no competing interests.

\section{REFERENCES}

1. Alonso J, Angermeyer MC, Bernert S, et al: Use of mental health services in Europe: results from the European Study of the Epidemiology of Mental Disorders (ESEMeD) project. Acta Psychiatrica Scandinavica 109:47-54, 2004

2. Verhaak PFM, Prins MA, Spreeuwenberg $P$, et al: Receiving treatment for common mental disorders. General Hospital Psychiatry 31:46-55, 2009

3. Verhaak PFM, van Beljouw IMJ, van Dijk CE: Policy Mental Health Care: Supporting Analyses [in Dutch]. Utrecht, Netherlands Institute for Health Services Research, 2010

4. Prins MA, Verhaak PFM, Bensing JM, et al: Health beliefs and perceived need for mental health care of anxiety and depression-the patients' perspective explored.

Clinical Psychology Review 28: 1038-1058, 2008

5. Churchill R, Khaira M, Gretton V, et al: Treating depression in general practice: factors affecting patients' treatment preferences. British Journal of General Practice 50:905-906, 2000 
Verhaak, P.F.M., Kamsma, H., Niet, A. van der. Mental health treatment provided by primary care psychologists in the Netherlands. Psychiatric Services: 2013, 64(1), 94-97

6. Cuijpers $P$, van Straten A, van Schaik A, et al: Psychological treatment of depression in primary care: a meta-analysis. British Journal of General Practice 59: e51-e60, 2009; doi 10.3399/bjgp09X395139

7. Bower P, Rowland N, Hardy R: The clinical effectiveness of counseling in primary care: a systematic review and meta-analysis. Psychological Medicine 33: 203-215, 2003

8. Harkness EF, Bower PJ: On-site mental health workers delivering psychological therapy and psychosocial interventions to patients in primary care: effects on the professional practice of primary care providers. Cochrane Database of Systematic Reviews 1:CD000532, 2009

9. van Dijk S, Knispel A, Nuijen J: Mental Health Care in Figures [in Dutch]. Utrecht, Netherlands, Trimbos Instituut, 2009

10. Codony M, Alonso J, Almansa J, et al: Perceived need for mental health care and service use among adults in Western Europe: results of the ESEMeD project.

Psychiatric Services 60:1051-1058, 2009

11. van Orden M, Hoffman T, Haffmans J, et al: Collaborative mental health care versus care as usual in a primary care setting: a randomized controlled trial. Psychiatric Services 60:74-79, 2009

12. Hilsenroth MJ, Ackerman SJ, Blagys MD, et al: Reliability and validity of DSM-IV axis V. American Journal of Psychiatry 157:1858-1863, 2000

\section{TABLE}

Multivariate logistic regression analysis of predictors of three outcomes among Dutch patients $(\mathrm{N}=51,473)$ treated by primary care psychologists in 2010

\begin{tabular}{|c|c|c|c|c|c|c|}
\hline \multirow[b]{3}{*}{ Variable } & \multirow{2}{*}{\multicolumn{2}{|c|}{$\begin{array}{l}\text { Regular conclusion } \\
\text { of treatment }\end{array}$}} & \multicolumn{4}{|c|}{ Clients with a regular treatment conclusion } \\
\hline & & & \multicolumn{2}{|c|}{$\begin{array}{l}\text { Improvement of } \\
>10 \mathrm{GAF} \text { points }\end{array}$} & \multicolumn{2}{|c|}{$\begin{array}{l}\text { Conclusion in } \\
\leq 8 \text { sessions }\end{array}$} \\
\hline & OR & $99 \% \mathrm{CI}$ & OR & $99 \% \mathrm{CI}$ & OR & $99 \% \mathrm{CI}$ \\
\hline Women (reference: men) & $1.08^{*}$ & $1.01-1.16$ & $1.15^{*}$ & $1.06-1.25$ & $.86^{*}$ & $.80-.93$ \\
\hline \multicolumn{7}{|l|}{ Age (reference: $0-18$ ) } \\
\hline $19-30$ & $.74^{*}$ & $.63-.88$ & $.81^{*}$ & $.66-.99$ & 1.06 & $.88-1.30$ \\
\hline $31-40$ & .83 & $.69-1.01$ & $.77^{*}$ & $.61-.97$ & .86 & $.69-1.08$ \\
\hline $41-50$ & .97 & $.80-1.17$ & $.76^{*}$ & $.60-.96$ & .82 & $.66-1.04$ \\
\hline $51-60$ & 1.16 & $.95-1.43$ & $.7^{*}$ & $.61-.98$ & .85 & $.67-1.08$ \\
\hline$\geq 61$ & $1.40^{*}$ & $1.03-1.91$ & $68^{*}$ & $.49-.95$ & .88 & $.63-1.22$ \\
\hline \multicolumn{7}{|l|}{ Marital status (reference: unmarried) } \\
\hline Married or cohabitating & $1.34^{*}$ & $1.23-1.47$ & 1.05 & $.95-1.18$ & 1.10 & $.99-1.22$ \\
\hline Divorced & 1.14 & $1.00-1.31$ & .89 & $.76-1.04$ & 1.10 & $.94-1.28$ \\
\hline Widowed & $1.36^{\circ}$ & $1.03-1.80$ & 1.08 & $.81-1.44$ & 1.20 & $.89-1.61$ \\
\hline \multicolumn{7}{|l|}{ Education (reference: low) } \\
\hline Middle & $1.34^{*}$ & $1.23-1.46$ & $1.26^{*}$ & $1.14-1.40$ & $.87^{\circ}$ & $.78-.96$ \\
\hline High & $1.52^{*}$ & $1.39-1.66$ & $1.32^{*}$ & $1.19-1.47$ & $.71^{*}$ & $.64-.79$ \\
\hline \multicolumn{7}{|l|}{ Not born in the Netherlands } \\
\hline (reference: born in the Netherlands) & $.63^{\circ}$ & $.56-.70$ & $.63^{*}$ & $.54-.74$ & 1.06 & $.92-1.24$ \\
\hline \multicolumn{7}{|l|}{$\begin{array}{l}\text { Employment status } \\
\text { (reference: employed) }\end{array}$} \\
\hline Self-employed & $.85^{\circ}$ & $.74-.97$ & .91 & $.79-1.05$ & .95 & $.83-1.10$ \\
\hline Unemployed or incapacitated & $.50^{\circ}$ & $.46-.55$ & .77 & $.68-.87$ & $.69^{*}$ & $.62-.78$ \\
\hline Pensioner & .84 & $.63-1.11$ & .82 & $.61-1.09$ & 1.30 & $.97-1.75$ \\
\hline Student & .94 & $.81-1.08$ & 1.03 & $.87-1.23$ & .89 & $.75-1.06$ \\
\hline \multicolumn{7}{|l|}{ Request for help ${ }^{b}$} \\
\hline Interpersonal problems & .98 & $.90-1.07$ & $.68^{*}$ & $.62-.75$ & $1.28^{*}$ & $1.17-1.41$ \\
\hline Work or school problems & $1.33^{\circ}$ & $1.17-1.51$ & 1.14 & $.99-1.30$ & .96 & $.85-1.09$ \\
\hline Depressive feelings & $.76^{*}$ & $.69-.83$ & 1.12 & $.99-1.26$ & $.69^{*}$ & $.61-.76$ \\
\hline Feeling anxious, nervous & $.89^{*}$ & $.80-.99$ & $1.15^{*}$ & $1.01-1.29$ & $.77^{*}$ & $.68-.86$ \\
\hline Adjustment problems & $1.37^{*}$ & $1.26-1.50$ & $1.21^{*}$ & $1.10-1.33$ & $1.19^{\circ}$ & $1.09-1.31$ \\
\hline Identity problems & .99 & $.84-1.16$ & $1.50^{*}$ & $1.23-1.82$ & .89 & $.75-1.08$ \\
\hline Psychosomatic problems & $.84^{\circ}$ & $.73-.99$ & 1.00 & $.82-1.21$ & .97 & $.80-1.17$ \\
\hline \multicolumn{7}{|l|}{ Sleeping, eating, sexual, } \\
\hline or impulse problems & $.69^{*}$ & $.56-.84$ & .80 & $.62-1.04$ & 1.04 & $.80-1.34$ \\
\hline Child problems & $1.53^{\circ}$ & $1.17-1.98$ & $1.40^{*}$ & $1.00-1.97$ & $.65^{\circ}$ & $.46-.92$ \\
\hline
\end{tabular}

a Corrected for initial Global Assessment of Functioning (GAF) score

${ }^{\mathrm{b}}$ Each help request was compared with all other requests (reference). * $\mathrm{p}<.001$ 